\title{
Pre-habilitation for patients awaiting total knee replacement in the United Kingdom National Health Service: A review of publicly facing information
}

\author{
Gareth Stephens $^{\mathrm{a}, *}$, Ahmed Maarabouni ${ }^{\mathrm{a}}$, Gemma Mansell $^{\mathrm{b}}$ and Chris Littlewood ${ }^{\mathrm{c}}$ \\ ${ }^{a}$ The Royal Orthopaedic Hospital, Birmingham, UK \\ ${ }^{\mathrm{b}}$ School of Psychology, College of Health and Life Sciences, Aston University, Aston Triangle, Birmingham, UK \\ ${ }^{\mathrm{c}}$ Department of Health Professions, Faculty of Health, Psychology \& Social Care, Manchester Metropolitan \\ University, Manchester, UK
}

Received 30 April 2021

Accepted 28 June 2021

\begin{abstract}
.
INTRODUCTION: Approximately 14,000-21,500 individuals per year are dissatisfied with the outcome of their Total Knee Replacement (TKR) in the UK National Health Service (NHS). National Institute of Clinical Excellence (NICE) guidelines recommend that future research should evaluate whether a 'full programme of pre-habilitation' can improve outcomes for patients awaiting TKR. The aim of this review was to describe current pre-habilitation practice for patients awaiting TKR in the UK NHS, to inform future research.

METHODS: Two reviewers independently undertook electronic searches for publicly available information sheets (PIS) from websites of UK NHS Trusts that included detail about pre-habilitation for patients awaiting TKR. One reviewer extracted data, and a second reviewer verified this.

RESULTS: Fifty PIS, nine information videos and one web page from 59 NHS Trusts were identified. NHS Trusts most commonly provide patients with advice on pre-operative rehabilitation via a single appointment, combined with a PIS (36/59; $61.0 \%)$. NHS Trusts use appointments, PIS and video to provide patients awaiting TKR with information regarding pain control $(46 / 58 ; 79.3 \%)$, exercise therapy $(46 / 58 ; 79.3 \%)$, what to expect on the day of surgery and in-patient stay $(58 / 58$; $100 \%)$, lifestyle interventions (27/58; 46.6\%), and adverse events (44/58; 75.9\%).

CONCLUSION: NHS Trusts commonly provided patients awaiting TKR with 'advice on pre-operative rehabilitation', however no NHS Trust provided a comprehensive programme of pre-habilitation. The results of this study will inform the development of a comprehensive, multi-modal pre-habilitation programme, to be tested in a future high-quality randomised controlled trial.
\end{abstract}

Keywords:

\section{Introduction}

In 2019 , more than 108,000 total knee replacements (TKR) were performed in the UK, costing the

\footnotetext{
*Corresponding author: Gareth Stephens, The Royal Orthopaedic Hospital NHS Trust, Physiotherapy Department, The Woodlands, Bristol Road South, Birmingham, B31 2AP, UK. Tel.: + 31 121 6854120; E-mail: Gareth.stephens@nhs.net.
}

NHS over $£ 700$ million [1]. TKR remains a successful intervention for many people with painful knee osteoarthritis. However, dissatisfaction rates following TKR are commonly reported at $13-20 \%$, which equates to approximately $14,000-21,500$ individuals per year [2,3]. Those who are dissatisfied following TKR, are likely to report persistent post-operative pain (often similar to pre-surgery), low levels of function and poor quality of life [4-6]. 
Pre-habilitation, targeting factors associated with poor outcomes is an approach used before many common surgeries (e.g., cardiovascular, oncology) as a way of improving outcomes post-surgery [7]. Pre-habilitation aims to promote healthy behaviours through needs-based prescription of exercise, lifestyle advice, nutrition support and psychological interventions [7, 8]. Traditionally, pre-habilitation for patients awaiting TKR has focussed on education and exercise [8]. Education strategies are used to address patient expectations as a means of improving post-operative satisfaction [7]. Exercise is commonly used to optimise pre-operative function as a way of improving post-operative pain and function and reducing post-operative complications [9]. However, recent systematic reviews have concluded that these traditional pre-habilitation programmes do not improve pain, function or length of stay in hospital following joint replacement $[9,10]$. One explanation for this is that there are a range of factors associated with poor outcomes following surgery including age, gender, comorbidities, expectations, pain, function, and mental health [11-16]. Dissatisfaction following surgery is therefore likely to be linked to a complex inter-play between many factors [5]. Given this, pre-habilitation approaches for patients awaiting TKR need to evolve (in keeping with pre-habilitation for patients awaiting cancer surgery) to include interventions which support patients with nutrition, medical optimisation, mental health, lifestyle factors (e.g., smoking) and expectations [17, 18].

Recent NICE guidelines made a strong recommendation for clinical practice, that 'advice on pre-operative rehabilitation' is provided for all patients awaiting TKR (delivered as a single appointment, individually or part of a group). The guidelines were unable to recommend a 'full programme of pre-habilitation' as clinical trials were too small and under-powered to recommend implementation [19]. However, the NICE concluded that there was a clear signal from the research that pre-habilitation has the potential to improve outcomes for patients undergoing TKR and hypothesised that a substantial, multi-dimensional package of pre-habilitation may improve outcomes for patients undergoing TKR [19]. NICE recommended that future research should develop and evaluate a 'full programme of pre-habilitation', able to support patients with needsbased support with lifestyle factors (such as smoking, alcohol consumption, dietary advice, and weight loss), activity levels (via exercise interventions) and mental health concerns (via counselling, cognitive behavioural interventions). This recommendation has been supported by two recent systematic reviews $[9,10]$.

To inform the development of a comprehensive pre-habilitation programme, we first wanted to understand what constitutes current practice in the UK NHS for patients awaiting TKR [20]. Therefore, the primary aim of this review of publicly facing information was to describe current pre-habilitation practice for patients awaiting TKR in the UK NHS. The secondary aim was to evaluate what models of prehabilitation are provided for patients awaiting TKR in terms of how they are delivered, the content of the interventions and the staffing groups they are delivered by. The final aim was to describe adherence with current NICE guidelines for pre-habilitation for patients awaiting TKR.

\section{Methods}

Two reviewers (AM and GS) undertook initial independent electronic searches of Google during January 2021, for publicly available patient information sheets (PIS) or information videos from websites of UK NHS Trusts. As this was a survey of publicly facing information, no NHS Trusts were directly contacted as part of the search strategy. The following search terms were used:

1. rehabilitation, knee replacement, nhs

2. physiotherapy, knee replacement, nhs.

3. pre-op, knee replacement, nhs

4. pre-habilitation, knee replacement, nhs

5. patient information, knee replacement, nhs

6. enhanced recovery, knee replacement, nhs

\subsection{Inclusion criteria}

Any PIS or information, provided by an NHS Trust for patients awaiting TKR, which provided patients with information aimed at improving their outcomes or expectation post-surgery. For example, advice on exercises, lifestyle changes or education aimed at influencing expectations.

\subsection{Exclusion criteria}

Data concerning pre-operative medical examinations used to determine anaesthetic risk were not extracted and analysed, as they were not relevant to the aims of this current study. 
The list of PIS were checked for duplications and duplicates were excluded. Searching continued until review of one full search page returned no relevant PIS as conducted in two other recent surveys of publicly facing information [21, 22]. Results of the separate searches were compared, and any disagreements resolved through discussion.

\subsection{Data extraction}

A data capture form was designed by the study team and piloted by two authors (AM and GS) on three PIS. Disagreements were discussed and final adaptations to the form were made. One reviewer (AM), then extracted data from the PIS and video links and populated the data capture form. All the extracted data was verified by a second reviewer (GS) and three disagreements (regarding exercise categorisation) were resolved through discussion.

\subsection{Statistical analysis}

Descriptive statistics were used to analyse the number of PIS and information videos that reported on pre-determined categories, established as part of the piloting of the data capture form. Data were described as a percentage of the total number of NHS Trusts surveyed (59), unless otherwise stated.

\section{Results}

A total of 78 web-links were clicked. Web-links either led directly to a PIS, or to an NHS Trust webpage. Once duplicate links were excluded (19), 50 PIS, nine information videos and one web page (providing limited information about a face-to-face intervention provided by one NHS Trust) from 59 NHS Trusts were included. Of the 50 PIS, 39 reported date of production, and $25(64 \%)$ of these were dated 2017 onwards (date range 2012 to 2020 ).

\subsection{PIS and Video information content}

The content from 50 PIS and nine information videos from 58 NHS Trusts were analysed to understand the information provided to patients awaiting TKR. Whilst no NHS Trust provided patients with supervised support with factors that may affect outcome, all Trusts provided patients with pre-operative information aimed at improving expectations and outcome, including pain control $(46 / 58 ; 79.3 \%)$, exercise therapy $(46 / 58 ; 79.3 \%)$, what to expect on the day of surgery and in-patient stay (58/58; $100 \%$ ), lifestyle interventions (e.g. smoking cessation, healthy eating) $(27 / 58 ; 46.6 \%)$, and possible adverse events $(44 / 58 ; 75.9 \%)$ associated with the surgery (e.g. deep venous thrombosis, infection).

\subsubsection{Pain control}

The majority of NHS Trusts $(46 / 58 ; 79.3 \%)$ provided patients with information regarding pain control. This typically included advice for patients to expect pain $(46 / 58 ; 79.3 \%)$ and swelling $(39 / 58$; $67.2 \%$ ) post-surgery. Patients were commonly advised on the analgesia that would be available to them on the ward $(43 / 58 ; 74.1 \%)$ and the use of ice $(32 / 58 ; 55.2 \%)$ to help them control their pain and swelling.

\subsubsection{Exercise therapy}

Patients were provided with information regarding post-operative exercise by $79.3 \%(46 / 58)$ of NHS Trusts. The outlined exercises commonly targeted quadriceps $(46 / 58 ; 79.3 \%)$ strengthening and improving range of motion of the operated knee $(45 / 58 ; 77.6 \%)$. Other commonly prescribed exercises included gastrocnemius $(29 / 58 ; 50 \%)$, gluteal $(14 / 58 ; 24.1 \%)$, hamstring $(14 / 58 ; 24.1 \%)$ and functional $(12 / 58 ; 20.7 \%)$ strengthening (exercises that replicated a daily task such as stepping up a step or standing from a chair). The majority (42/58) of NHS trusts provided patients with a combination on written texts and images of their post-operative exercises. Four NHS Trusts provided the information via video only.

\subsubsection{The day of surgery and in-patient stay}

All NHS Trusts provided information on what to expect on the day of surgery $(58 / 58 ; 100 \%)$, during the inpatient stay $(58 / 58 ; 100 \%)$ and at the point of discharge $(58 / 58 ; 100 \%)$. Patients were advised about what to bring into hospital with them $(58 / 58 ; 100 \%)$, that they would see a physiotherapist to help them mobilise within a day of surgery $(53 / 58 ; 91.4 \%)$ and the expected length of stay in hospital $(52 / 58 ; 89.7 \%)$.

\subsubsection{Lifestyle interventions}

Less than half of the NHS Trusts $(27 / 58 ; 46.6 \%)$ advised patients about lifestyle changes that could improve their outcomes after surgery. Advice on the benefits of exercise and activity $(21 / 58 ; 36.2 \%)$ and cutting down / stopping smoking $(21 / 58 ; 36.2 \%)$ were the most common lifestyle interventions advised. 


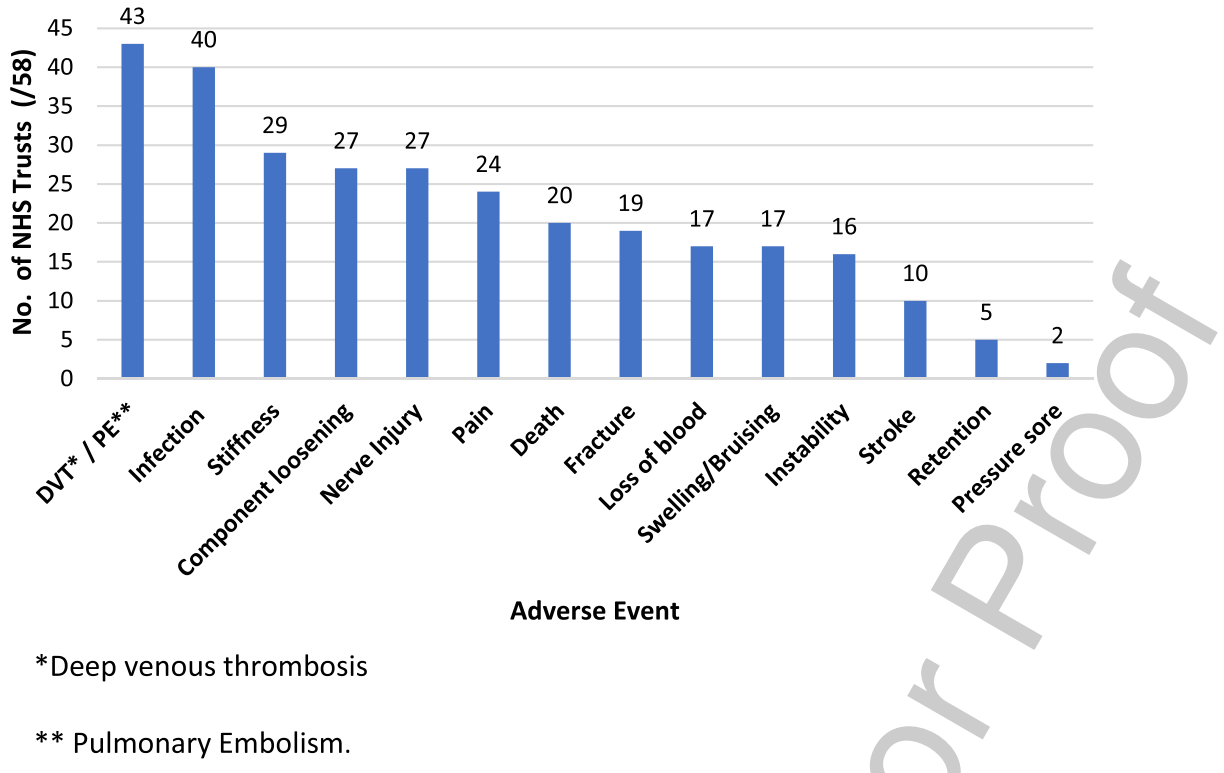

Fig. 1. Number of NHS Trusts providing patients with information about specific adverse events.

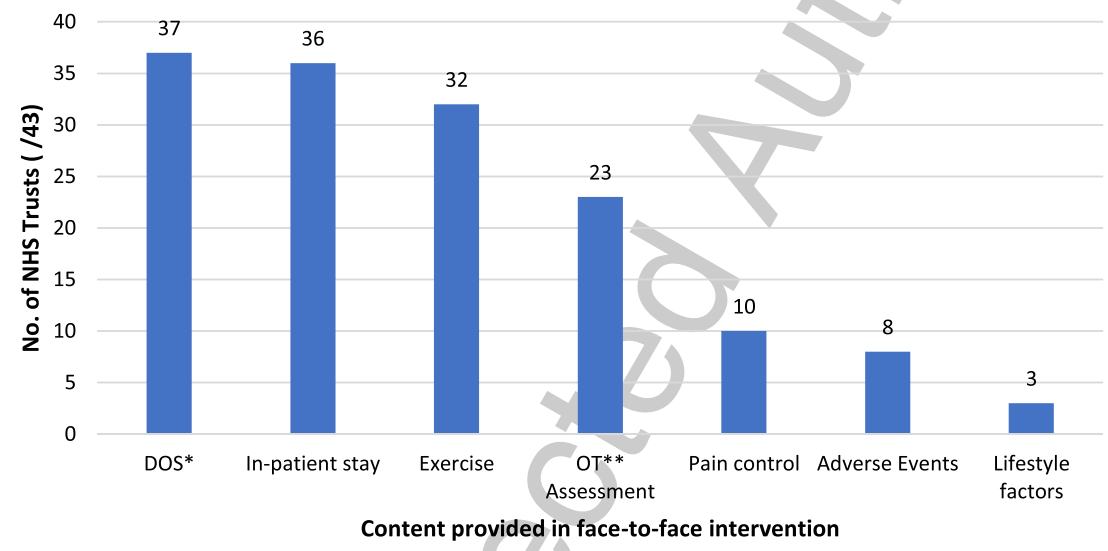

*Information regarding the day of surgery

**Occupational Therapy

Fig. 2. Information provided as part of face-to-face session.

Almost one third of NHS Trusts advised patients about a balanced diet $(18 / 58 ; 31.0 \%)$ and advice on maintaining a healthy weight $(18 / 58 ; 31.0 \%)$. Other lifestyle interventions included alcohol intake (13/58; $22.4 \%$ ) and the possible benefits of a positive mindset $(4 / 58 ; 6.9 \%)$.

\subsubsection{Adverse events}

Potential adverse events following TKR were described by $75.9 \%$ (44/58) of NHS Trusts in their patient facing information. The adverse events most 238 patients were informed about are reported in Fig. 1. $\quad 239$

\subsection{Face-to-face appointments}

The data provided in the PIS and video information does not provide definitive information regarding the content and staffing of the face-to-face appointments, however the information in Fig. 2 provides a summary the data that was obtainable. The information 
provided to patients in the face-to-face appointments appeared to be no different to those described in the PIS and video information (Fig. 2). However, 74.4\% (32/43) of NHS Trusts providing face-to-face interventions described using staff members outside of physiotherapy such as occupational therapy (24/43), nurses (22/43), doctors (8/43), and dietitians (2/43). More than one staff group were described as being present in $60.5 \%(26 / 43)$ of NHS Trusts providing a face-to-face appointment.

\subsection{Adherence with the NICE guidelines}

The majority of NHS Trusts adhered to the NICE guidelines $(42 / 59 ; 71.2 \%)$ by providing 'advice on pre-operative rehabilitation' via a single appointment. This appointment was most often supplemented with either a PIS $(36 / 59 ; 61.0 \%)$, video information $(5 / 59 ; 8.4 \%)$, or both $(1 / 59 ; 1.7 \%)$. Other modes of delivery included provision of only a PIS $(13 / 59 ; 22.0 \%)$, video information $(3 / 59 ; 5.1 \%)$, or appointment $(1 / 59 ; 1.7 \%)$. No NHS Trust provided patients awaiting TKR with more than one face-toface pre-habilitation appointment.

\section{Discussion}

This paper reports the findings of a review of publicly facing information for patients awaiting TKR in the UK NHS. The majority of NHS Trusts $(42 / 59 ; 71.2 \%)$ adhered to the NICE guidelines by providing patients with 'advice on pre-operative rehabilitation' via a single appointment. Most commonly, this appointment was combined with a PIS $(36 / 59 ; 61.0 \%)$. These appointments and PIS were used to provide patients with information regarding pain control, exercise therapy, what to expect on the day of surgery and in-patient stay, lifestyle interventions, and possible adverse events associated with the surgery. No NHS Trust in this review provided patients with information regarding a 'full programme of pre-habilitation' as outlined in the research recommendations in the NICE guidelines [19].

A 'full programme pre-habilitation' addressing factors associated with poor outcome following surgery could make people better able to deal with the possible complications, promote understanding and engagement with postoperative rehabilitation, and prepare the person better for existing with a replaced joint [19]. NICE research recommendations (2020) suggest a comprehensive pre-habilitation programme for patients awaiting TKR could include exercise interventions, psychological assessment (with counselling or cognitive therapy), weight control (via dietary support and advice), pain control (via pain medication review, exercise and education), interventions to maximise independence (via assessment of activities of daily living and equipment provision), and lifestyle advice (via support with smoking and alcohol reduction or cessation).

This review highlights that as part of current standard care, patients awaiting TKR are educated on optimisation of their pain control, what to expect during their in-patient care, and potential adverse events. In addition, patients are also asked to consider several complex behavioural changes (e.g., smoking cessation, healthy eating, weight loss, increased physical activity and exercise) to reduce the impact of lifestyle factors associated with poorer outcomes. Individuals who are obese, smoke or drink alcohol excessively, are at higher risk of poor outcomes and post-operative complications following TKR [23-25]. Almost half of the NHS Trusts in this review advised patients awaiting TKR to consider lifestyle changes however, achieving meaningful lifestyle and behaviour change is a complex process for many people [26, 27]. Explaining the risk of continuing with a behaviour which may be detrimental to their long-term health, does not lead to significant changes in behaviour, and thus current approaches used within the NHS are unlikely to achieve meaningful change [28]. Patients may require substantial support to achieve meaningful change in their lifestyle habits than is currently on offer from routine NHS services [28].

The NICE guidelines highlighted the detrimental impact that mental health problems can have on outcomes following joint replacement [19]. Very few NHS Trusts provided patients with information regarding their mental health. Mental health problems, such as depression and anxiety, are consistently demonstrated as prognostic for poor outcomes following TKR [5, 25, 29]. It is unclear whether physiotherapists can successfully deliver interventions which directly target anxiety and depression [30]. However, physiotherapy interventions can improve the quality of life of patients with mental health disorders [31]. Interventions which may directly, or indirectly improve pain, self-efficacy, self-esteem, and physical activity have the potential to reduce the impact of mental health conditions [32]. A full programme of pre-habilitation would need to screen patients for mental health conditions and offer 
interventions which improve outcomes such as counselling or cognitive behavioural therapy [19].

It is a research priority to design and evaluate a full programme of pre-habilitation for patients awaiting TKR [19]. This review of publicly facing information reveals that this full programme of pre-habilitation would involve a large deviation from current practice with NHS. It would require significant resources and a multi-disciplinary approach. Future research in this field will require development of a complex intervention, to be tested in a future randomised controlled trial.

\subsection{Strengths and limitations}

The strengths of this study include the large number of retrieved PIS from a range of NHS Trusts across the United Kingdom. Two reviewers undertook the searches and data extraction, in line with current best practice. Therefore, it is sufficient to provide an overview of current practice, answer the research question and guide future research.

The limitations of this reviews of this nature are that they are reliant on the quality of the information provided on the PIS and NHS Trust website. It therefore remains unclear how well the 59 NHS Trusts reflect current practice across the UK NHS. Not all NHS Trusts provide online information for patients and keep the information up to date.

\section{Conclusion}

The majority of NHS Trusts adhere to the current NICE guidelines regarding pre-habilitation for patients awaiting TKR, by providing 'advice on preoperative rehabilitation' via an appointment. NICE guidelines suggest that it is a research priority to evaluate whether a comprehensive multi-modal prehabilitation programme (with the potential of offer patients the necessary support required to adopt these behaviour changes) can improve outcomes for patients undergoing TKR. This review highlights that this would involve a significant change from current NHS practice and potentially, large amounts of NHS resource. Therefore, a comprehensive programme of intervention development and evaluation (via a highquality randomised controlled trial) is required to evaluate whether a full programme of pre-habilitation can improve outcomes for patients undergoing TKR.
Acknowledgments

The Royal Orthopaedic Hospital Research and Development department for supporting the corresponding author.

\section{Conflict of interest}

The authors have no conflict of interest to report.

\section{References}

[1] National Joint Registry. NJR 17th Annual Report 2020 2020; Available from: https://reports.njrcentre.org.uk/ downloads

[2] Kahlenberg CA, Nwachukwu BU, McLawhorn AS, Cross MB, Cornell CN, Padgett DE. Patient Satisfaction After Total Knee Replacement: A Systematic Review. HSS J. 2018;14(2):192-201.

[3] NHS. NHS improvement: National payment tariff system. 2020; Available from: https://improvement.nhs.uk/ resources/national-tariff/\#h2-202021-national-tariffpayment-system

[4] Beswick AD, Wylde V, Gooberman-Hill R, Blom A, Dieppe $\mathrm{P}$. What proportion of patients report long-term pain after total hip or knee replacement for osteoarthritis? A systematic review of Prospective studies in unselected patients. BMJ Open. 2012;2(1):1-12.

[5] Gunaratne R, Pratt DN, Banda J, Fick DP, Khan RJK, Robertson BW. Patient Dissatisfaction Following Total Knee Arthroplasty: A Systematic Review of the Literature. J Arthroplasty [Internet]. 2017;32(12):3854-60. Available from: http://dx.doi.org/10.1016/j.arth.2017.07.021

[6] Jones CA, Beaupre LA, Johnston DWC, Suarez-Almazor ME. Total joint arthroplasties: Current concepts of patient outcomes after surgery. Clin Geriatr Med. 2005;21(3 SPEC. ISS.):527-41.

[7] Durrand J, Singh SJ, Danjoux G. Prehabilitation. Clin Med (Northfield Il). 2019;17(6):458-64.

[8] Jahic D, Omerovic D, Tanovic AT, Dzankovic F, Campara MT. The Effect of Prehabilitation on Postoperative Outcome in Patients Following Primary Total Knee Arthroplasty. Med Arch (Sarajevo, Bosnia Herzegovina). 2018;72(6):439-43.

[9] Dennis J, Wylde V, Gooberman-Hill R, Blom AW, Beswick AD. Effects of presurgical interventions on chronic pain after total knee replacement: a systematic review and meta-analysis of randomised controlled trials. BMJ Open. 2020;10(1).

[10] Moyer R, Ikert K, Long K, Marsh J. The Value of Preoperative Exercise and Education for Patients Undergoing Total Hip and Knee Arthroplasty: A Systematic Review and Meta-Analysis. JBJS Rev. 2017;5(12):e2.

[11] Gandhi R, Razak F, Tso P, Davey JR, Mahomed NN. Greater perceived helplessness in osteoarthritis predicts outcome of joint replacement surgery. J Rheumatol. 2009;36(7): 1507-11.

[12] Hilton ME, Gioe T, Noorbaloochi S, Singh JA. Increasing comorbidity is associated with worsening physical function and pain after primary total knee arthroplasty. BMC 
Musculoskelet Disord [Internet]. 2016;17(1):421. Available from: https://www.ncbi.nlm.nih.gov/pubmed/27717340

[13] Judge A, Arden NK, Cooper C, Kassim Javaid M, Carr AJ, Field RE, et al. Predictors of outcomes of total knee replacement surgery. Rheumatology (Oxford). 2012; 51(10):1804-13.

[14] Lungu E, Desmeules F, Dionne CE, Belzile EL, Vendittoli P-A. Prediction of poor outcomes six months following total knee arthroplasty in patients awaiting surgery. BMC Musculoskelet Disord. 2014;15:299.

[15] Murphy BP d'S., Dowsey MM, Spelman T, Choong PFM. The impact of older age on patient outcomes following primary total knee arthroplasty. Bone Joint $\mathrm{J}$ [Internet]. 2018;100-B(11):1463-70. Available from: https://doi.org/ 10.1302/0301-620X.100B11.BJJ-2017-0753.R6

[16] Pinto PR, McIntyre T, Ferrero R, Almeida A, Araújo-Soares V. Risk Factors for Moderate and Severe Persistent Pain in Patients Undergoing Total Knee and Hip Arthroplasty: A Prospective Predictive Study. PLoS One. 2013;8(9).

[17] Bolshinsky V, Li MHG, Ismail H, Burbury K, Riedel B, Heriot A. Multimodal Prehabilitation Programs as a Bundle of Care in Gastrointestinal Cancer Surgery: A Systematic Review. Dis Colon Rectum. 2018;61(1):124-38.

[18] Bousquet-Dion G, Awasthi R, Loiselle SÈ, Minnella EM, Agnihotram R V., Bergdahl A, et al. Evaluation of supervised multimodal prehabilitation programme in cancer patients undergoing colorectal resection: a randomized control trial. Acta Oncol (Madr) [Internet]. 2018;57(6):849-59. Available from: https://doi.org/10.1080/0284186X.2017. 1423180

[19] NICE. Joint replacement (primary): Hip, knee and shoulder joint replacement: evidence review for preoperative rehabilitation [Internet]. 2020. Available from: https://www. nice.org.uk

[20] MRC. Medical Research Council. Developing and Evaluating complex interventions. 2006; Available from: https:// mrc.ukri.org/search-results/?keywords=Developing+and+ evaluating+complex+interventions\&siteid $=\mathrm{mrc}$

[21] Littlewood C, Morgan M, Pitt L, Moffatt M, Edwards P, Davies R, et al. Rehabilitation following shoulder arthroplasty in the United Kingdom National Health Service: A survey of publicly facing information. Musculoskeletal Care. 2020;18(3):359-64.

[22] Rohun J, May P, Littlewood C. Rehabilitation following proximal humeral fracture in the UK National Health Service: A survey of publicly facing information. Musculoskeletal Care. 2020;(October):1-6.
[23] Harris AHS, Reeder R, Ellerbe L, Bradley KA, Rubinsky AD, Giori NJ. Preoperative alcohol screening scores: Association with complications in men undergoing total joint arthroplasty. J Bone Jt Surg - Ser A. 2011;93(4):321-7.

[24] Matharu GS, Mouchti S, Twigg S, Delmestri A, Murray DW, Judge A, et al. The effect of smoking on outcomes following primary total hip and knee arthroplasty: a population-based cohort study of 117,024 patients. Acta Orthop. 2019;90(6):559-67.

[25] Yang HY, Losina E, Lange JK, Katz JN, Collins JE. Longitudinal Trajectories of Pain and Function Improvement Following Total Knee Replacement. ACR open Rheumatol. 2019;1(5):308-17.

[26] Burke V, Milligan RA, Beilin LJ, Dunbar D, Spencer M, Balde E, et al. Clustering of health-related behaviors among 18-year-old Australians. Prev Med (Baltim). 1997;26 (5 Pt 1):724-33.

[27] Koelewijn-Van Loon MS, Van Steenkiste B, Ronda G Wensing M, Stoffers HE, Elwyn G, et al. Improving patient adherence to lifestyle advice (IMPALA): A clusterrandomised controlled trial on the implementation of a nurse-led intervention for cardiovascular risk management in primary care (protocol). BMC Health Serv Res. 2008;8: $1-15$.

[28] French DP, Cameron E, Benton JS, Deaton C, Harvie M Can Communicating Personalised Disease Risk Promote Healthy Behaviour Change? A Systematic Review of Systematic Reviews. Ann Behav Med. 2017;51(5):718-29.

[29] Stephens G, Nightingale P, Mylogiannakis P, Suokas A. Do early patient reported outcome measures post total knee arthroplasty predict poor outcomes (the early PROMPT study)? Physiother Pract Res. 2020;41(2):109-20.

[30] Torales J, Barrios I, Almirón M, De la Cueva R. Physiotherapy in the treatment of anxiety disorders. Int $\mathrm{J}$ Cult Ment Health [Internet]. 2017;10(3):298-9. Available from: https://doi.org/10.1080/17542863.2017.1303075

[31] Richardson CR, Faulkner G, McDevitt J, Skrinar GS Hutchinson DS, Piette JD. Integrating physical activity into mental health services for persons with serious mental illness. Psychiatr Serv. 2005;56(3):324-31.

[32] Rosenbaum S, Sherrington C, Tiedemann A. Exercise augmentation compared with usual care for post-traumatic stress disorder: a randomized controlled trial. Acta Psychiatr Scand. 2015;131(5):350-9. 